[in press at Neuron]

\title{
A framework to address cognitive biases of climate change
}

\author{
Jiaying Zhao ${ }^{1,2}$ and $\mathrm{Yu} \mathrm{Luo}^{1}$ \\ ${ }^{1}$ Department of Psychology, University of British Columbia \\ ${ }^{2}$ Institute for Resources, Environment and Sustainability, University of British Columbia
}

Please address correspondence to:

Jiaying Zhao Department of Psychology Institute for Resources, Environment and Sustainability University of British Columbia Vancouver, B.C., Canada, V6T 1Z4

Email: jiayingz@psych.ubc.ca 


\begin{abstract}
We propose a framework that outlines several predominant cognitive biases of climate change, identifies potential causes, and proposes debiasing tools, with the ultimate goal of depolarizing climate beliefs and promoting actions to mitigate climate change.
\end{abstract}

Keywords: decision making, cognition, behavior change, polarization, debias 


\section{Introduction}

Climate change is an urgent crisis facing humanity. To effectively combat climate change, we need concerted efforts not only from policymakers and industry leaders to institute top-down structural changes (e.g., policies, infrastructure) but also from individuals and communities to instigate bottom-up behavior changes to collectively reduce greenhouse gas emissions. To this end, psychology has offered useful insights on what motivates people to act on climate change, from social psychology investigating the underlying beliefs and attitudes of climate change, to cognitive psychology uncovering the attentional, perceptual, and decision processes of climate actions, and more recently neuroscience pinpointing the neural circuitry on motivated cognition. Insights gained from these fields have started to generate interventions to shift beliefs and promote behavior change to mitigate climate change.

In the search for behavioral climate solutions, a stubborn challenge remains. That is, a sizeable portion of the public still hold disbelief on climate change and refuse to take actions, despite the fact that the vast majority of climate scientists have reached a consensus on anthropogenic causes of climate change. In fact, public opinion on climate change has polarized along party lines in North America over the last few decades. According to a recent poll by the Pew Research Center in 2021, 40\% of Republicans remain skeptical about the role of human activities in climate change, where most Democrats have reached an agreement on the anthropogenic causes.

To address climate hesitancy and polarization, it is essential to understand the cognitive biases that distort the mental representation of climate change and subsequently lead to faulty climate beliefs and prevent climate actions. Here, we propose a framework that first outlines the predominant cognitive biases that can give rise to disbelief and skepticism on climate change and 
perpetuate polarization, then identifies the causes of the biases, and proposes debiasing tools to mitigate the biases, reshape climate beliefs, and promote climate actions (see Figure 1).

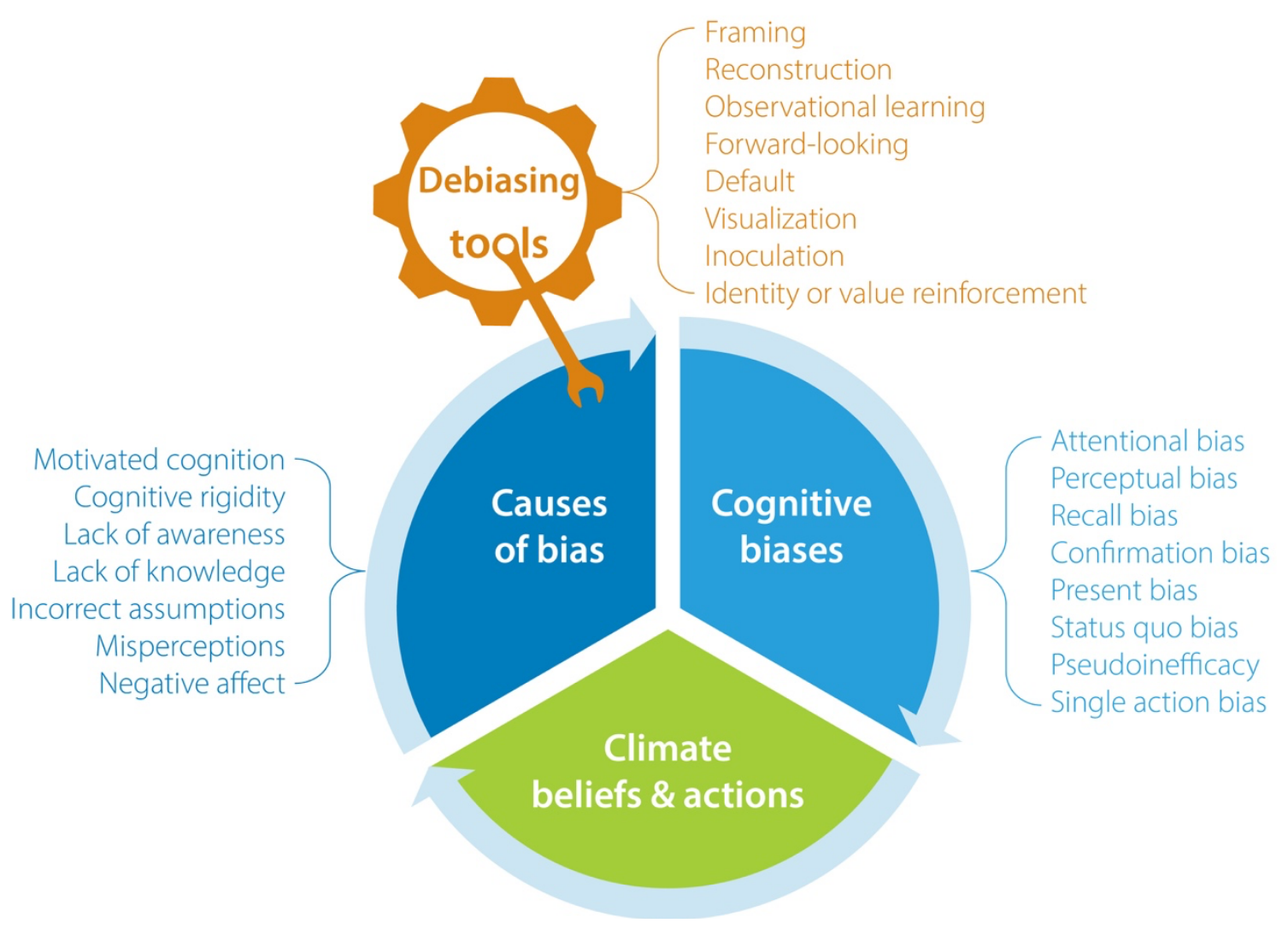

Figure 1: A framework on cognitive biases of climate change

We illustrate this framework with an example. An attentional bias away from climate change evidence among conservative individuals can be driven by motivated cognition stemming from prior beliefs, and this bias can attenuate their concerns about climate change and fortify the resistance to reduce carbon emissions. The reduced concern and inaction can further reinforce motivated cognition, forming a positive feedback loop. A potential debiasing tool to address the attentional bias is to visualize global temperature in relation to greenhouse gas emissions from human activities as opposed to natural causes (e.g., solar activities). This tool can draw attention to the human contributions to climate change relative to natural factors, highlighting the need to reduce human-caused carbon emissions. In the following sections, we discuss the cognitive 
biases, potential causes, and debiasing tools in detail (see Table 1). Most biases identified so far

involve an under-representation of the urgency and severity of climate change, while a few

involve an over-representation (e.g., heightened attention to climate change) that can lead to eco-

anxiety and sense of hopelessness (e.g., pseudoinefficacy).

Table 1: A summary of the cognitive biases, causes, and debiasing tools

\section{Cognitive biases}

Attentional bias

- attending to climate change

information differently depending on

political orientations

Perceptual bias

- inaccurate perceptions of ingroup

norms and outgroup norms

- negative footprint illusion

Recall bias

- falsely recalling climate change to be less serious, which is associated with higher skepticism of climate change

\section{Confirmation bias}

- seeking information that confirms prior beliefs, or discounting information that contradicts prior beliefs

\section{Present bias}

- priority given to people and objectives that are close in terms of time and space than more distant ones

\section{Status quo bias \\ - unwillingness to change the status quo because any change involves effort and uncertainty}

\section{Pseudoinefficacy}

- a false sense of inefficacy of individual actions not making an appreciable contribution to solving a large overall problem

\section{Single action bias}

- perceiving a reduced risk of climate change after taking one climate action

\section{Causes of bias}

Motivated cognition

- information processing

being shaped by personal

goals and needs

Cognitive rigidity

- inability to adapt to a new environment or update prior beliefs given new information

Lack of awareness

- no awareness of the urgency and severity of climate change

Lack of knowledge

- no knowledge about the causes or consequences of climate change, or what actions to take

\section{Incorrect assumptions}

- inaccurate understandings of the drivers of climate change

\section{Misperceptions}

- underestimations of carbon footprints associated with individual actions

\section{Negative affect}

- reduced negative emotion after taking one climate action
Debiasing tools

Framing

- aligning climate communication

with peoples' ideologies and values

Reconstruction

- reconstructing accurate

representations of social norms

\section{Observational learning}

- acquiring attitudes, norms, and facts by observing the behavior of others or watching videos

Forward-looking - generating arguments for forwardlooking options or considering the legacy for future generations

Default

- making climate-friendly options the default

\section{Visualization}

- highlighting human contributions to climate change as opposed to natural causes

\section{Inoculation}

- exposing people to forewarning and using preemptive refutation to protect them from misinformation

Identity or value reinforcement

- reinforcing one's identity and values to promote subsequent climate actions 


\section{Cognitive biases}

This section describes several key cognitive biases on climate change discovered in prior literature, provides examples associated with each bias, and explains the downstream consequences of the biases on climate beliefs and actions.

Recent studies revealed an attentional bias of climate change, where people attend to climate information differently depending on their political orientation or prior beliefs about climate change (Luo and Zhao, 2021). In a series of eye-tracking studies, people who selfidentified as more liberal focused more on the rising phase of a global temperature curve relative to the flatter phase of the curve, while those who self-identified as more conservative focused more on the flatter phase. This selective attention is also shown in other studies using an attentional blink paradigm and an attention cueing paradigm, where people who had greater concerns about climate change prioritized their attention toward climate-related words (e.g., carbon) or images (e.g., melting ice) over neutral ones. Attention away from climate information in conservatives can reinforce their climate disbelief and inaction, while attention toward climate information in liberals or the concerned can elevate their belief and level of concern of climate change, thus contributing to polarization.

In addition to selective attention, a perceptual bias arises from selective perception where people misperceive in-group and out-group norms by overweighing the voices and views from a few members of in-group or out-group members. This selective perception exaggerates the degree of support for a given climate policy from in-group members or the degree of opposition from out-group members, thus creating a false sense of polarization. A related memory bias is when people falsely recall the severity of climate change, and those who recall climate change to be less severe tend to show stronger skepticism and disbelief of climate change. The motivated 
recall can serve to protect and justify the previously held skepticism and disbelief. Apart from selective perception, another perceptual bias is the negative footprint illusion where people perceive a lower carbon footprint of a combination of conventional items and environmentally friendly items than the carbon footprint of the conventional items alone.

A well-known cognitive bias is confirmation bias, which is the tendency to actively seek information that confirms prior beliefs (e.g., reading news articles that challenge climate change among conservatives), or to discount information that contradicts prior beliefs. This bias creates a barrier for shifting conservatives' position on climate change when the intervention only presents pro-climate change information. A recent paper revealed the neural mechanism of this bias, which suggests that the neural representation of information strength is weakened when others' opinions oppose one's own (Kappes et al., 2020). In other words, during information processing the brain tends to disregard the strength of opposing evidence but incorporates the strength of confirming evidence. This bias provides one explanation of how some people remain skeptical about anthropogenic climate change despite overwhelming evidence by ignoring the strength of the scientific consensus on climate change.

A decision bias that presents another barrier for climate mitigation is present bias, which is the tendency to prioritize objectives or tasks that are temporally and spatially closer over the ones that are more distant (Weber, 2017). A consequence of this bias is that people tend to overvalue the immediate costs of climate mitigation policies (e.g., carbon tax) and undervalue the future benefits (e.g., greenhouse gas emission reductions). For individual climate actions, this bias can deter people from purchasing energy efficient appliances due to the high upfront cost despite equally high (if not higher) future energy savings. A related decision bias is the status quo bias, which suggests that people prefer to stick with the status quo rather than choosing a 
new option to minimize effort and uncertainty associated with the change. This bias hinders efforts to promote climate actions that require changes to people's current lifestyles even though the changes are beneficial over the long term.

Another prominent decision bias is pseudoinefficacy, which is a false sense of inefficacy of individual actions that are perceived to be not making an appreciable contribution to solving a large overall problem (Västfjäll et al., 2015). If people believe that individual climate actions and efforts cannot meaningfully mitigate climate change, they may feel hopeless and will not take any action. This bias is fostered by a harmful narrative that propagates climate change as a global challenge only requiring large-scale government and industry changes, with individual behavior change at the margins. This narrative overlooks the importance of individual actions from consumers and citizens who are often the driving force behind policy change and social movements, and deters the public from taking meaningful climate actions.

Among people who have initiated climate mitigation behaviors, they may suffer from single action bias (Truelove et al., 2014), a tendency to perceive a reduced risk of climate change after taking one single climate action, although a combination of actions is more effective. Single action bias can lead to negative spillover effects where adopting one pro-environmental behavior reduces the probability of taking additional actions. Moreover, after engaging in one climate mitigation action, people may experience a heightened moral self-image, and consequently, are less likely to perform another climate action, known as moral licensing.

\section{Causes of bias}

Most research to date has focused on identifying the biases of climate change, but relatively less work has examined the causes of the bias. In order to minimize cognitive biases, it 
is critical to understand the underlying driving forces. In this section, we offer some hypotheses on the potential factors that can contribute to the cognitive biases.

A primary cause of the biases is motivated cognition, which is that prior motivations (e.g., personal goals, needs) can influence information processing at multiple stages, resulting in attentional, perceptual, recall, and confirmation biases. For example, motivations to identify with and belong to a certain political group can determine how an individual processes climate change information depending on whether the information is consistent with the group's ideologies, although such causal evidence is still lacking (most evidence so far is correlational). Another factor driving motivated cognition could be prior beliefs that serve to maintain consistency in opinions rather than protecting partisan identities. Recent neuroscience studies have shown that motives can modulate neural systems associated with attentional control and low-level perception by shifting attention and perception toward motivationally consistent information and away from inconsistent ones (Hughes and Zaki, 2015).

In addition to motivated cognition, cognitive rigidity can be a driver of some biases. Cognitive rigidity is defined as a cognitive trait characterized by an inability to adapt to a new environment or respond dynamically to neuropsychological tasks and is suggested to correlate with extreme political actions and more ideological and dogmatic worldviews (Zmigrod and Goldenberg, 2021). Cognitive rigidity can also cause a failure to update prior beliefs given new information. In the case of climate change, cognitively rigid individuals may fail to change their beliefs when presented with climate change evidence, giving rise to confirmation bias or status quo bias. They may also experience problems adapting to climate change as adaptation requires changes in their environment (e.g., moving to a new location with a lower risk of flooding). 
Other causes include lack of awareness of the urgency and severity of climate change, lack of knowledge of the drivers or consequences of climate change or what actions to take, incorrect assumptions of what causes climate change, and misperceptions of the carbon footprint associated with individual actions. The lack of awareness of the urgent need to mitigate climate change can contribute to present bias and status quo bias due to a false sense of contentment. The lack of knowledge has been shown to correlate with less concern about climate change, which can contribute to status quo bias.

Conservatives often adopt the view that climate change is caused by natural factors instead of human activities, and this incorrect assumption can contribute to confirmation bias and pseudoinefficacy. Moreover, people tend to underestimate the carbon emissions associated with daily individual actions regardless of their political orientations and climate concerns (Camilleri et al., 2019). This underestimation can cause pseudoinefficacy by minimizing the climate impact of their own actions, and also present bias by devaluing the carbon savings of switching to climate friendlier alternatives. Finally, the single action bias can be caused by the temporary alleviation of negative affect after performing one climate action (Truelove et al., 2014).

\section{Debiasing tools}

Identify potential causes of the bias can inform the design of debiasing tools to increase the acceptance of anthropogenic climate change and the adoption of climate change mitigation behaviors. In this section, we propose several debiasing tools to address the underlying causes.

There are a number of tools to address motivated cognition. A commonly used tool is framing, which involves aligning climate communication with peoples' ideologies and values. For example, religious leaders (e.g., Pope Francis) who are concerned about climate change can promote environmental actions among conservatives via shared religious faith. A recent field 
experiment showed that Republican elites associating their concerns of climate change with economy, national security, faith, and conservative values, increased Republican individuals' understanding of the occurrence, causes, and consequences of climate change (Goldberg et al., 2021). Another tool is to reconstruct an accurate representation of the social norms of the group by exposing people to the actual views on in-group and out-group members on climate change, or by encouraging people to communicate with more in-group and out-group members to get a representative view of the group. A third tool is observational learning which is a process of acquiring beliefs, norms, and facts by observing the behavior of others or watching videos.

To address cognitive rigidity, forward-looking techniques can be used that involve generating arguments for forward-looking options or considering the legacy for future generations. This tool can evoke personal reflections on how they want to be remembered by future generations (Weber, 2017). Another tool is to use default which involves making climatefriendly options the status quo, which reduces the effort and scrutinization of potential risks during the decision process.

One tool to increase the knowledge of climate change and to correct the incorrect assumptions is to use visualization techniques to highlight human contributions to climate change as opposed to natural causes over a long timescale. We should note that highlighting climate change evidence alone may backfire, as in a previous study conservatives were less likely to take climate actions when the rising phase of the global temperature curve was made salient (Luo and Zhao, 2021). Visualization thus must target the specific assumption that was incorrect or information of climate change that was not known before, rather than reiterating what is already known, to avoid possible backfiring. Visualizing existing and future damages caused by climate change can increase the awareness of the urgency and severity of climate 
change. To solve the underestimation problem of carbon footprint, visualization techniques can be used to present accurate and digestible carbon footprint information of individual actions (e.g., one roundtrip from New York to London) to rebuild an accurate understanding of the climate impact of individual actions. This information can also help people decide which actions to take to mitigate climate change (Camilleri et al., 2019).

The lack of knowledge and awareness of climate change also makes people susceptible to misinformation. A meta-analysis showed that interventions that induced skepticism on climate change had a stronger effect on attitudes than those that aimed to increase belief, suggesting that belief in climate change is harder to build but easier to demolish (Rode et al., 2021). To combat misinformation, inoculation can be used by first exposeing people to warnings of false claims and then preemptively refuting these claims to protect them from misinformation. For example, after seeing a message of scientific consensus on climate change, participants were first warned about a false claim in a misinformation campaign, and then read a statement that refuted the false claim with a reiteration of the consensus among climate scientists. These participants maintained positive views toward the consensus after being exposed to misinformation but not those who were not inoculated against misinformation (van der Linden et al., 2017).

Lastly, a debiasing tool for the single action bias is reinforcing the connection between climate actions and a person's identity or values by reflecing on the how the behavior is related to their values, identity, or views on how people in society should behave. Such intervention has been shown to increase the adotption of subsequent climate actions (Sparkman et al., 2021).

\section{A call for future research}

The current framework is only a start of identifying cognitive biases, the causes, and the debiasing tools. It is far from a comprehensive list. We call for future research in (1) discovering 
cognitive biases of climate change, (2) identifying the root causes of the biases, (3) developing the corresponding debiasing tools, (4) testing the tools in randomized field experiments, and (5) tracking both short-term and long-term impact on the bias and the downstream changes in climate beliefs (e.g., personal importance and concerns) and climate actions (e.g., reducing carbon emissions, voting). While this framework heavily focuses on the contributions from social and cognitive psychology, more research on the underlying neural mechanisms of the cognitive biases and debiasing tools is needed. We urge cognitive, behavioral, and neuroscientists to enrich the current framework to collectively discover the remedy for cognitive biases of climate change, with the ultimate goal of depolarizing beliefs and promoting actions to conquer the climate crisis. 


\section{Declaration of Interests}

The authors declare no competing interests.

\section{Acknowledgments}

We thank Nigel Deans and two anonymous reviewers for providing helpful comments. This work was supported by the Canada Research Chairs program (to JZ) and Social Sciences and Humanities Research Council Doctoral Fellowship (to YL). 


\section{References}

Camilleri, A.R., Larrick, R.P., Hossain, S., and Patino-Echeverri, D. (2019). Consumers underestimate the emissions associated with food but are aided by labels. Nat. Clim. Chang. 9, 53-58.

Goldberg, M.H., Gustafson, A., Rosenthal, S.A., and Leiserowitz, A. (2021). Shifting Republican views on climate change through targeted advertising. Nat. Clim. Chang. 8-9.

Hughes, B.L., and Zaki, J. (2015). The neuroscience of motivated cognition. Trends Cogn. Sci. $19,62-64$.

Kappes, A., Harvey, A.H., Lohrenz, T., Montague, P.R., and Sharot, T. (2020). Confirmation bias in the utilization of others' opinion strength. Nat. Neurosci. 23, 130-137.

van der Linden, S., Leiserowitz, A., Rosenthal, S., and Maibach, E. (2017). Inoculating the Public against Misinformation about Climate Change. Glob. Challenges 1, 1600008.

Luo, Y., and Zhao, J. (2021). Attentional and perceptual biases of climate change. Curr. Opin. Behav. Sci. 42, 22-26.

Rode, J.B., Dent, A.L., Benedict, C.N., Brosnahan, D.B., Martinez, R.L., and Ditto, P.H. (2021). Influencing Climate Change Attitudes in the United States: A Systematic Review and Meta-Analysis. J. Environ. Psychol. 76, 101623.

Sparkman, G., Attari, S.Z., and Weber, E.U. (2021). Moderating spillover: Focusing on personal sustainable behavior rarely hinders and can boost climate policy support. Energy Res. Soc. Sci. 78, 102150.

Truelove, H.B., Carrico, A.R., Weber, E.U., Raimi, K.T., and Vandenbergh, M.P. (2014). Positive and negative spillover of pro-environmental behavior: An integrative review and theoretical framework. Glob. Environ. Chang. 29, 127-138. 
Västfjäll, D., Slovic, P., and Mayorga, M. (2015). Pseudoinecacy and the Arithmetic of Compassion. In Numbers and Nerves: Information, Emotion, and Meaning in a World of Data., S. Slovic, and P. Slovic, eds. (Oregon State University Press), pp. 42-52.

Weber, E.U. (2017). Breaking cognitive barriers to a sustainable future. Nat. Hum. Behav. 1, 13. Zmigrod, L., and Goldenberg, A. (2021). Cognition and Emotion in Extreme Political Action: Individual Differences and Dynamic Interactions. Curr. Dir. Psychol. Sci. 30, 218-227. 